\title{
NANOPARTICLES CARRYING NATURAL PRODUCT FOR DRUG DELIVERY
}

\author{
Suhui Ni \\ Department of Pharmacy, China Pharmaceutical University, Nanjing, PR China, 211100
}

Article Info: Received 16 March 2017; Review Completed 22 April 2017; Accepted 05 May 2017, Available online 15 May 2017

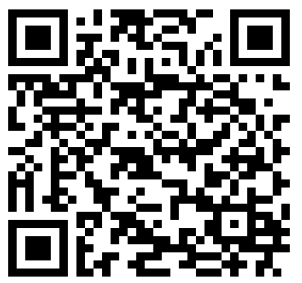
Cite this article as:

$\mathrm{Ni} \mathrm{S}$, Nanoparticles carrying natural product for drug delivery, Journal of Drug Delivery and Therapeutics. 2017; 7(3):73-75

DOI: http://dx.doi.org/10.22270/jddt.v7i3.1425

*Address for Correspondence:

Suhui Ni, Department of Pharmacy, China Pharmaceutical University, Nanjing, PR China, 211100

Email: nisuhui2014@qq.com

\begin{abstract}
Camptothecin, Doxorubicin, Paclitaxel, Vincristine, and Etoposide are the naturally occurring anti-cancer drugs that are analyzed in this analysis. It is found that these compounds can be developed as nanomedicine. It has been determined from analyses that nanoparticles are useful in targeted drug delivery to address some of the innate issues. However, there are certain limitations that are found in nanomedicine. There is a need for more research to develop effective drugs in the future. A more detailed analysis of the same has been done in the following.
\end{abstract}

Keywords: Camptothecin, Doxorubicin, Paclitaxel, Vincristine, Etoposide, Targeted drug delivery, mechanism of action, toxicity, limitations

\section{INTRODUCTION}

Nanoparticles have more surfaces to volume ratio. These nanoparticles are found to revolutionize drug delivery. ${ }^{1}$ This is because the drugs target the viruses and tumors and they are found to not affect the healthy tissue., ${ }^{2,3}$ The bioavailability of the particles is found to be the reason for the Nanomedicine to gain precedence.

Natural product is one of the best sources for procuring drugs and drug leads. ${ }^{4-6}$ From the year 1940 onwards, 131 drugs out of 175 small molecule anticancer drugs are found to be based on the natural product. ${ }^{7}$ This is owing to the fact that the natural products are found to have diversity in their physical structure and have chemical diversity. $40 \%$ of the chemical scaffolding is found in natural products. ${ }^{8,9}$ One of the most important reasons for considering natural products is that they are optimized evolutionarily as a drug-like molecule..$^{10-12}$ This is based on the natural products and these drugs are found to occupy the same molecular volume. ${ }^{13}$
Some of the nanoparticles that have been developed from the natural products are discussed in the following.

\section{Camptothecin}

Camptothecin is found to plant alkaloid that is initially isolated from Camptotheca acuminate. Camptothecin is found in the bark, leaves, and stem in the Chinese happy tree. Camptotheca acuminate is a native breed to China. ${ }^{14,15}$ These are considered to be important for the anticancer properties of Camptothecin. ${ }^{16-18}$ This is considered to be the traditional medicine that has been used for many centuries. This Camptothecin compound is found to have an important role in curing of a number of diseases including Nanomedicine treatment for cancer. This Camptothecin compound is found to halt the cell during the $\mathrm{S}$ phase mitosis. It is found to have a nanomolar potency to address the issues of cytotoxicity against the human tumor cell lines such as HT29, LOX to name a few. In combination with the TNF, it is found to induce apoptosis. A number of feasible drugs that are developed from the Camptothecin analogs are used in the development of anti-cancer drugs. Some of them are 
topotecan and irinotecan. ${ }^{19,20}$ These have been commercially sold as anti-cancer drugs and in chemotherapy ${ }^{21}$ These compounds are now being used in Nanomedicine. They are found to have long circulation half-lives and localize the tumor cells. The nanoparticles address the tumor cells only and release the CPT and cause them to disassemble the individual polymer chains. ${ }^{22}$ These are found to be smaller and cleared renally. IT-101 is one such drug that is currently investigated. Hence, this compound with a lot of potentials is found to originate from natural sources. Its complete potential in nanomedicine is yet to be utilized completely.

\section{Etoposide}

Etoposide is a compound that is extracted from the Podophyllum hexandrum. It is difficult to grow this plant. Owing to this, it was found that they can transfer the enzyme producing gene from $\mathrm{P}$. hexandrum to Nicotiano benthamiana. This is a tobacco-like plant that is easier to grow. The engineered plant is found to produce only nanograms of etoposide aglycone as a precursor to Etoposide The prolific growth of the yeast cell can lead to better production. Etoposide is anticancer chemotherapy drug. This drug is a plant alkaloid and it functions as a topoisomerase II inhibitor. In the current times, it is used as a semisynthetic derivative of podophyllotoxin that is found to exhibit antitumor activity. The Etoposide inhibit DNA is found and is found to form a complex with the topoisomerase II and DNA. ${ }^{23-25}$ The complex is used to induce the break in the double stranded DNA and is found to prevent repair of the topoisomerase II binding. The accumulated then breaks the DNA into the mitotic phase of cell division. This eventually causes apoptosis. This then enters into the G2 and $\mathrm{S}$ phases of cell cycle. Currently, the feasibility of administering the medicine in the form of nanoparticles is being studied. ${ }^{26}$ The complete potential of the Etoposide is yet to be harnessed completely.

\section{Vincristine}

Vincristine is a vinca alkaloid that is isolated from the Vinca Rosea. It has been marketed under many brand names. These are found in many formulations. This is used in the treatment of acute leukemia, Hodgkin's disease, malignant lymphoma and acute erythraemia. This vincristine sulfate is used as a part of the polychemotherapy as it stems from the lack of significant bone-marrow suppression. It has a unique clinical toxicity. This inhibits the mitosis at the metaphase stage that stems from the interaction with the tubulin. They interfere with the amino acid, glutathione metabolism, calmodulin-dependent $\mathrm{Ca} 2+-$ or transport the ATPase activity or impact the cellular respiration and lipid biosynthesis. Currently, the lower solubility of the compound is causing a detrimental impact. This has impeded the process of nanomedicine. A number of clinical trials are being undertaken to understand the impact of the compound. ${ }^{27}$

\section{Paclitaxel}

Paclitaxel is one of the compounds for cancer drug in the United States. This is found to be derived from the bark of the Taxus brevifolia. It is used in the treatment of Kaposi sarcoma and a number of cancers.(28-30) In this, the complex of $\alpha, \beta$ tubulins subunits along with Paclitaxel is used to correct the issues in mitotic spindle assembly, cell division, and chromosome segregation. ${ }^{31,32}$ This medicinal compound is used in Nanomedicine. The encapsulation of Paclitaxel is found to be biodegradable and has non-toxic Nano-delivery systems that reduce the toxicity of the company and increases the circulation half-life. It has found to yield a better result. This is also found in improved pharmacokinetic profiles. Paclitaxel along with the albumin-bound nanoparticles is used as a commercial medicine to treat the metastatic breast cancer. This drug is currently being used for the treatment of breast cancer on a commercial scale. This is one of the few nanomedicine drugs that have managed to address all the issues. ${ }^{33}$ There are a number of novel paclitaxel nanoparticles that are current being analyzed.

\section{Doxorubicin}

Doxorubicin is found to be extracted from the anthracycline antibiotic that is cytotoxic and isolated from the Streptomyces peucetius cultures. These compounds are found to bind to the nucleic acid based on the specific intercalation of the planar antracyanine nucleus along with the double-helical structure of the DNA. It is found to impede the growth of the Cancer cells in the body. ${ }^{34}$ The doxorubicin blocks the function of the enzyme topoisomerase 2 . This is needed by the cancer cells to proliferate. ${ }^{35-37}$ Some of the factors that are considered in this situation are the efficacy of the drug based on the exposure of the tissue. The commerce and the toxicity are some of the other factors that are considered. $^{38}$ There has been an improved comprehension of the heterogeneity of clinical cancers and its biological factors. There is a transition that has been observed in the case of formulation-driven research to it being disease driven development. The nanomedicine needs to be developed and the potential needs to be fully harnessed.

\section{SUMMARY}

Camptothecin, Doxorubicin, Paclitaxel, Vincristine, and Etoposide are some of the naturally occurring anti-cancer drugs that are discussed in this analysis. The mechanism of action, sources of the compounds and the limitation during commercial production of the compounds are discussed. From this, a number of insights are gained. These are natural medicines and they are found to be optimized evolutionarily as a drug-like molecule that makes it feasible for the commercial production of the compounds. These medicines are mostly administered as chemotherapy drugs. These medicines are found to have the potential to be administered as nanomedicine into the body. However, its complete potential is yet to be harnessed. Each of these five compounds is found to be naturally occurring and has a number of properties to impede the growth of the anti-cancer cells. In the case of nanomedicine, there can be targeted drug delivery and the toxicity of the medicine can be prevented in this process. There are a number of ongoing clinical trials that are being undertaken to commercially produce the 
compounds on a large scale. To conclude, the complete potential of the naturally occurring compounds that can be used as nanomedicines is yet to be harnessed.

\section{REFERENCES}

1. Hamidi M, Azadi A, and Rafiei P. Hydrogel nanoparticles in drug delivery. Advanced drug delivery reviews. 2008, 60: 1638-1649

2. Cheng X. Developing organic and inorganic nanomedicine for cancer therapy. Journal of Drug Delivery and Therapeutics. 2017, 7(3): 1-4 DOI: 10.22270/jddt.v7i2.1367

3. Cheng $X$, and Lee $R$ J. The role of helper lipids in lipid nanoparticles (LNPs) designed for oligonucleotide delivery. $A d v$ Drug Deliv Rev. 2016, 99: 129-137; doi: 10.1016/j.addr.2016.01.022.

4. Cheng X, Liu Q, Li H, Kang C, Liu Y, Guo T, et al. Lipid Nanoparticles Loaded with an Antisense Oligonucleotide Gapmer Against Bcl-2 for Treatment of Lung Cancer. Pharm Res. 2017, 34: 310-320; doi: 10.1007/s11095-016-2063-5.

5. Duan Y, Pei K, Cai H, Tu S, Cheng X, Zhang Z, et al. Strategy of integrated evaluation on treatment of traditional Chinese medicine as 'interaction of system to system' and establishment of novel fuzzy target contribution recognition with herb-pairs, a case study on Astragali Radix-Fructus Corni. Molecular and Cellular Endocrinology. 2016, 434: 219-237

6. Duan Y, Pei K, Cai H, Tu S, Zhang Z, Cheng X, et al. Bioactivity evaluation-based ultra high-performance liquid chromatography coupled with electrospray ionization tandem quadrupole-time-offlight mass spectrometry and novel distinction of multisubchemome compatibility recognition strategy with Astragali Radix-Fructus Corni herb-pair as a case study. Journal of pharmaceutical and biomedical analysis. 2016, 129: 514-534

7. Liu Z, Jiao Y, Wang Y, Zhou C, and Zhang Z. Polysaccharidesbased nanoparticles as drug delivery systems. Advanced drug delivery reviews. 2008, 60: 1650-1662

8. Sun Y, and Kang C. Self-Assembly of Peptides into Hydrogel. Journal of Organic \& Inorganic Chemistry. 2016,

9. Sun Y, Kang C, Liu F, and Song L. Delivery of Antipsychotics with Nanoparticles. Drug Development Research. 2016, 77: 393 399

10. Sun Y, Kang C, Yao Z, Liu F, and Zhou Y. Peptide-Based Ligand for Active Delivery of Liposomal Doxorubicin. Nano LIFE. 2016, 6: 1642004

11. Sun Y, Kang C, Zhang A, Liu F, Hu J, Zhong X, et al. Codelivery of dual-drugs with nanoparticle to overcome multidrug resistance. European Journal of BioMedical Research. 2016, 2: $12-18$

12. Waller A P, George M, Kalyanasundaram A, Kang C, Periasamy $\mathrm{M}$, Hu K, et al. GLUT12 functions as a basal and insulinindependent glucose transporter in the heart. Biochimica et Biophysica Acta (BBA)-Molecular Basis of Disease. 2013, 1832: 121-127

13. Soppimath K S, Aminabhavi T M, Kulkarni A R, and Rudzinski W E. Biodegradable polymeric nanoparticles as drug delivery devices. Journal of controlled release. 2001, 70: 1-20

14. Hsiang Y-H, Hertzberg R, Hecht S, and Liu L. Camptothecin induces protein-linked DNA breaks via mammalian DNA topoisomerase I. Journal of Biological Chemistry. 1985, 260: $14873-14878$

15. Li Q-Y, Zu Y-G, Shi R-Z, and Yao L-P. Review camptothecin: current perspectives. Current medicinal chemistry. 2006, 13: 2021-2039

16. Kang C, Qin J, Osei W, and Hu K. Regulation of protein kinase $\mathrm{C}$-epsilon and its age-dependence. Biochemical and Biophysical Research Communications. 2017, 482: 1201-1206

17. Kang C, Sun Y, Wang M, and Cheng X. Nanosized camptothecin conjugates for single and combined drug delivery. European Journal of BioMedical Research. 2016, 2: 8-14

18. Kang C, Sun Y, Zhu J, Li W, Zhang A, Kuang T, et al. Delivery of Nanoparticles for Treatment of Brain Tumor. Current Drug Metabolism. 2016, 17: 745-754

19. Li H, Cheng X, Liu Y, Lee Y B, Kim D J, Ahn C-h, et al. Folate receptor-targeted lipid coated albumin nanoparticles (F-LCAN) for therapeutic delivery of RX-0201 (Archexin®), an antisense oligonucleotide against Akt-1. 2016,

20. Li H, Quan J, Zhang M, Yung B C, Cheng X, Liu Y, et al. LipidAlbumin Nanoparticles (LAN) for Therapeutic Delivery of Antisense Oligonucleotide against HIF-1alpha. Mol Pharm. 2016, 13: 2555-2562; doi: 10.1021/acs.molpharmaceut.6b00363.

21. Chen Y, Bian Y, Sun Y, Kang C, Yu S, Fu T, et al. Identification of 4-aminoquinoline core for the design of new cholinesterase inhibitors. PeerJ. 2016, 4: e2140

22. Davis M E. Design and development of IT-101, a cyclodextrincontaining polymer conjugate of camptothecin. Advanced drug delivery reviews. 2009, 61: 1189-1192

23. Guo X K, Sun H P, Shen S, Sun Y, Xie F L, Tao L, et al. Synthesis and evaluation of gambogic acid derivatives as antitumor agents. Part III. Chemistry \& biodiversity. 2013, 10 73-85

24. Liu Z-1, Zhang R-m, Meng Q-g, Zhang X-c, and Sun Y. Discovery of new protein kinase CK2 inhibitors with 1, 3-dioxo2, 3-dihydro-1 H-indene core. MedChem Comm. 2016, 7: 13521355

25. Sun H, Zhu J, Chen Y, Sun Y, Zhi H, Li H, et al. Docking Study and Three- Dimensional Quantitative Structure- Activity Relationship (3D- QSAR) Analyses and Novel Molecular Design of a Series of 4- Aminoquinazolines as Inhibitors of Aurora B Kinase. Chinese Journal of Chemistry. 2011, 29: 1785-1799

26. Qian W-Y, Sun D-M, Zhu R-R, Du X-L, Liu H, and Wang S-L. $\mathrm{pH}$-sensitive strontium carbonate nanoparticles as new anticancer vehicles for controlled etoposide release. Int J Nanomed. 2012, 7: 5781-5792

27. Wang Y, Dou L, He H, Zhang Y, and Shen Q. Multifunctional nanoparticles as nanocarrier for vincristine sulfate delivery to overcome tumor multidrug resistance. Molecular pharmaceutics. 2014, 11: 885-894

28. Liu F, Sun Y, Kang C, and Zhu H. Pegylated Drug Delivery Systems: From Design to Biomedical Applications. Nano LIFE. 2016, 6: 1642002

29. Qiao H, Fang D, Chen J, Sun Y, Kang C, Di L, et al. Orally delivered polycurcumin responsive to bacterial reduction for targeted therapy of inflammatory bowel disease. Drug Delivery. 2017, 24: 233-242

30. Song L, Kang C, Sun Y, Huang W, Liu W, and Qian Z. Crocetin Inhibits Lipopolysaccharide-Induced Inflammatory Response in Human Umbilical Vein Endothelial Cells. Cellular Physiology and Biochemistry. 2016, 40: 443-452

31. XU S-h, Chen K, CHEN M-l, ZHOU P-p, HE G-w, CUI Y-j, et al. Dynamic expression of AQP4 in early stageof ischemia/reperfusion rats and cerebral edema. Chinese Pharmacological Bulletin. 2016: 1433-1441

32. Xue X, Zhao N-Y, Yu H-T, Sun Y, Kang C, Huang Q-B, et al Discovery of novel inhibitors disrupting HIF-1 $\alpha$ /von HippelLindau interaction through shape-based screening and cascade docking. PeerJ. 2016, 4: e2757

33. Shin J-Y, Yang Y, Heo P, Lee J-C, Kong B, Cho J Y, et al. pHresponsive high-density lipoprotein-like nanoparticles to release paclitaxel at acidic $\mathrm{pH}$ in cancer chemotherapy. Int $J$ Nanomedicine. 2012, 7: 2805-2816

34. Han R, Sun Y, Kang C, Sun H, and Wei W. Amphiphilic dendritic nanomicelle-mediated co-delivery of 5-fluorouracil and doxorubicin for enhanced therapeutic efficacy. Journal of Drug Targeting. 2017, 25: 140-148

35. Yang Z, Xie J, Zhu J, Kang C, Chiang C, Wang X, et al Functional exosome-mimic for delivery of siRNA to cancer: in vitro and in vivo evaluation. Journal of Controlled Release. 2016, 243: $160-171$

36. Yao Z, Sun Y, and Kang C. Structure and Self-Assembly of Multicolored Naphthalene Diimides Semiconductor. Nano LIFE. 2016, 6: 1642007

37. Zhong X, Sun Y, Kang C, and Wan G. The theory of dielectrophoresis and its applications on medical and materials research. European Journal of BioMedical Research. 2017, 2: 7 11

38. Du J-Z, Du X-J, Mao C-Q, and Wang J. Tailor-made dual pHsensitive polymer-doxorubicin nanoparticles for efficient anticancer drug delivery. Journal of the American Chemical Society. 2011, 133: 17560-17563 